\title{
Improved Segmentation algorithm using PSO and K-means for Basal Cell Carcinoma Classification from Skin Lesions
}

\author{
Komal Sharma, Sanjay Madaan
}

\begin{abstract}
Skin carcinoma has been sighted as one of the prevalent forms of carcinomas, specifically amongst Caucasian offspring and pale-skinned population. Basal Cell Carcinoma $(B C C)$ is a malevolent skin carcinoma and its classification in earlier stage is a biggest issue. Whilst curable with early detection, only extremely skilled specialists are likely to recognize the disease accurately from skin lesions Dermoscopic images. Since expertise has limited contribution, an automatized system capable of classifying disease can be helpful in saving lives, reducing unnecessary biopsies, and extra costs. On the way to achieve this objective, we proposed a BCC classification model that unifies recent advances in deep learning with Artificial Neural Network (ANN) structure, creating hybrid algorithm of K-means segmentation with Particle Swarm Optimization (PSO) that are capable of segmenting accurate skin lesions region from dermoscopy images, as well as examining the detected region and neighboring tissues for BCC. The proposed system is evaluated using the largest publicly accessible standard skin lesions dataset of dermoscopic images, containing BCC and Non-BCC images. When the evaluation parameters of proposed work are contrasted with a couple of other top-of-the-line techniques, the proposed technique accomplishes superior performance of $97.9 \%$ with respect to area under the curve (AUC) in distinguishing BCC from benignant lesions only through the extricated Speeded Up Robust Features (SURF).
\end{abstract}

Index Terms: Artificial Neural Network (ANN), Computer Assisted Dermoscopy, Pattern recognition, Particle Swarm Optimization (PSO), Skin Lesion, Skin Lesion Segmentation, Speeded up Robust Features (SURF) analysis on Medical Image.

\section{INTRODUCTION}

Malignant Basal Cell Carcinoma (BCC) is amongst the most speedily growing carcinoma across the globe. It is estimated by the American Academy of Dermatology Association that 95,830 more cases of BCC will be discovered in U.S in 2019. Further, as per the Stanford University Researchers (2018) about 2 million new cases of BCCs are detected yearly. At present, in spite of some highly developed remedial techniques; in particular radiation and immunotherapy are progressively coupled with surgical procedures. Such inconsistencies stimulate the necessity of sensible diagnostics and timely treatment of malignant $\mathrm{BCC}$ for saving lives of people.

Revised Manuscript Received on July 10, 2019

Komal Sharma, Dept. of CSE, University Institute of Engineering, Chandigarh University, Gharuan, Mohali, India

Sanjay Madaan, Dept. of CSE, University Institute of Engineering, Chandigarh University, Gharuan, Mohali, India.
Skin covers the majority part of the body acquiring an area of 20 square feet. Its structure is shown in figure 1. Skin shields us from microorganisms, aids in regulating the core temperature of the body and sanctions the senses of touching, warmth and coldness.

The two fundamental layers of human skin are:

1. Epidermis and

2. Dermis

Epidermis being layered squamous epithelia acts as a shelter in defiance of external influences. The coloring of skin is developed by cells named Melanocytes, responsible for producing Melanin pigment. Melanocytes situated in Epidermis comprises of four kinds of cells:

* Keratinocytes: $95 \%$ of the epidermis cells are keratinocytes and are the impelling cause of constant revival of the skin.

* Melanocytes: Are the dendrite cells located in the base layering of the epidermis. Responsible for delivering packs of melanin to the neighboring keratinocytes to give skin \& hair its color.

* Langerhans cells: Are dendritic cells similar to Melanocytes but performs detection of extraneous matter infiltrated the epidermis and hand over them to nearby lymph nodes.

* Merkel cells: Possibly originated from keratinocytes, they serve as mechanosensory recipients responding to touch.

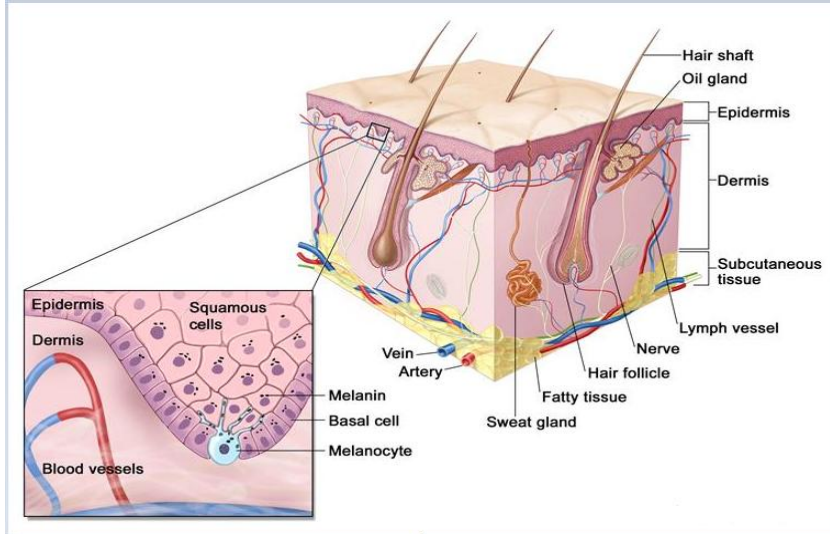

Fig. 1: Anatomy of the skin [1]

In the medical science, Dermoscopy is a most popular in vivo non intrusive imaging device that functions using polarization assisting skin specialists in investigating pigmentary skin lesions according to a feature set of morphologies. 
Although dermoscopy has shown increased analytical accuracy comparing to the predictable criteria, suitable interpretation of dermoscopic skin lesion images is usually time-consuming, more complex, and inclined to experience from inter and intra-observer variabilities. Consequently, computerized methods have already been developed to support dermatologists for enhancing their efficiency and objectiveness of visual understanding of dermoscopic images for prognostics model to predict diseases and their classification. Automatically segmenting skin lesion from the surrounding skin is an indispensable phase in computer based analysis of dermoscopic images. However, this assignment is not inconsequential because skin lesion usually has a wide variety of appearance in outline, dimension and color along with dissimilar types of human skin and their textures. Whilst some skin sores have uneven and blurry borders, and in several cases the contrast of lesion and the neighboring area of skin is pretty low. In additional case, artifacts and intrinsic features of human skin, viz hairs, blood vessel and space bubbles can make the automated segmentation of particular lesion more challenging, as illustrated in Fig. 2.

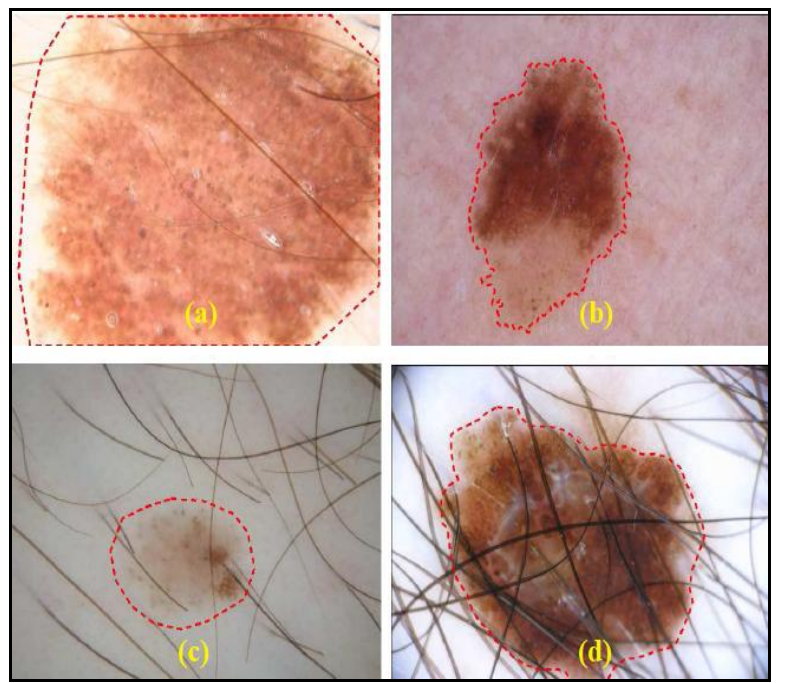

Fig. 2: Dermoscopic images with automated lesion segmentation. (a) bulky size of lesion; (b) uneven and blurry border; (c) lower contrast with respect to the surrounding skin; and (d) lesion with hair.

This paper presents an improved approach for prediction and classification of BCC from dermoscopy images using an Artificial Neural Network (ANN) and their comparison with existing trends. Specifically, in section 2, we present the literature survey (background survey) of existing work for detecting and segmenting vascular structures of skin lesions. The architecture of proposed work is described in the section 3. The simulation result is cover in section 4 and we conclude with discussions on current challenges and future trends in section 5 .

\section{BACKGROUND SURVEY}

This section presents the survey of existing work based upon the segmentation of vascular structures of skin lesions for disease classification using different techniques.
Pegah Kharazmi et al. [2] proposed an automatized recognition \& segmenting of vascular structures of lesions in skin dermoscopic images with an application to diagnose BCC. They first decomposed the skin dermoscopic image through Independent Component Analysis (ICA) in two components i.e. Hemoglobin \& Melanin. Further by means of k-means clustering clustered the hemoglobin component in three regions viz. normal, pigmentary \& erythematous. Next, they applied shape filters to the erythematous cluster at various scales. A vessel mask was then produced through global thresholding. In addition, they then specified and extricated a group of 12 features to feed in Random forest classifier. The outcomes exhibited superior performance of $96.5 \%$ over other top-of-the-line methods in distinguishing between benign lesions and BCC by making use of the extricated features.

Lequan $\mathrm{Yu}$ et al. [3] Introduced a novel approach for identifying Melanoma by making maximum use of profound Convolutional Neural Networks (CNNs). To get the most out of the deep networks, they suggested a group of schemes to assure effectual training and learning in line with finite training data. To begin with, they applied residual learning for dealing with challenges like overfitting \& degradation that often shows up once the network gets deeper. By doing so, they ascertain their networks advantaging from the execution gains obtained as a result of the increased network depth. Afterwards, they built a Fully Convolutional Residual Network (FCRN) for precisely segmenting skin lesion \& continued to strengthen its ability by embodying a multi-scale integration scheme. Ultimately, they constituted a two-tier structure by unifying the introduced FCRN for segmenting the lesion \& other profound residual networks for classification task. This system permits the classifying network to extricate better characteristics \& distinct features in accordance with segmented image in lieu of complete dermoscopic image. Experimental findings indicated the considerable performance gains of the developed framework.

Yading Yuan et al. [4] presented a totally automated technique for segmenting skin lesions by put to use 19-layer deep CNNs trained from one extreme to the other \& doesn't require any previous knowledge of the data. They proposed a group of schemes to assure efficacious \& efficient learning with finite training data. In addition to that, they designed a loss function on the basis of Jaccard distance for eradicating the requirement of sample reweighting. They assessed the degree of efficiency \& generalizing ability of the proposed system on two publicly accessible repositories. Result of experiments has shown that the proposed technique excelled the other top-of-the-line algorithms. This method is sufficiently general \& just requiring minimal pre-processing \& post-processing which provides it an opportunity for being endorsed in a number of different medical imaging segmentation tasks. 
N. C. F. Codella et al. [5] proposed a system which amalgamated recent advances in deep learning technology with Machine learning techniques, developing a group of methods capable of performing skin lesion segmentation, along with examining the identified portion $\&$ tissues around for detecting Melanoma. They assessed the proposed system through largest commonly accessible benchmark data collection of dermoscopic images comprising 900 training images and 379 testing images. New top-of-the-line performance was attained, resulting into betterment of the area under ROC curve and increased accuracy and specificity.

Fengying Xie et al. [6] designed a novel technique for classifying melanocytic tumors in the type of being benign or malignant tumor by analyzing the digitally acquired dermoscopic images. This technique comprises of three stages: first of all, lesions were extricated by employing a self-generating neural network (SGNN) [6]; secondly, characteristics representing color of tumor, its texture \& border were extricated \& finally, the objects of lesion were classified by making use of a classifier in accordance with a neural network ensemble model [6]. To cope up with the challenge that often comes up in clinical stances, novel border features were proposed capable of effectually distinguishing border irregularities of complete as well as incomplete lesions. To attain enhanced performance, they designed a classifier that unites back propagation neural networks along with fuzzy neural networks. Two different dermoscopic databases comprising images of Xanthous as well as Caucasian races were used for carrying out the experiments. The findings revealed that accuracy of classification has significant improvement as a result of using both, the newly proposed border features \& the classifier model.

Euijoon Ahn et al. [7] suggested saliency detection utilizing the reconstruction errors taken from an exiguous representative model together with new background detection could be furthermore precise in distinguishing the lesion from its neighboring regions. They also proposed a Bayesian framework which well outlines the border \& shape of the lesion. They valuated the proposed approach on two publically available datasets having 1100 dermoscopy images \& versus other traditional \& top-of-the-line unsupervised lesion segmenting approaches, as well as the top-of-the-line unsupervised salience detection approaches. The outcomes proved that the suggested method segments the lesion more precisely and vigorously when compared with other approaches.

C. Benazzi et al. [8] reviewed the fundamental and clinical prospects of angiogenesis placing an emphasis on the significance of comparative biology for the understanding of angiogenesis \& amalgamation of these system models in view of the forthcoming medicinal chemistry. From the survey they concluded that investigations under way shall valuate the highly effective fusion of inhibitor of angiogenesis with other targeted and/or traditional therapies for improving the medical results.

B. Cheng et al. [9] investigated a biologicly stimulated reinforcement learning method in an adaptive critic design structure for applying action-dependent heuristic dynamic programming (ADHDP) to perform segmentation on the basis of estimated features by using a number of lesion contrast varieties for promoting the segmentation task. Lesion segmentation outcomes for ADHDP were then compared with multilayer perception backpropagation neural networks. Experimental findings brought forth a highly distinctive accuracy of $84.6 \%$ using ADHDP method thereby providing a significant improvement of $8.03 \%$ over a standard multilayer perception technique. They chose detection of BCC over vessel detection for the reason that it has probable direct medical applications.

Based on the survey we conclude some important point which helps to short out existing problem. Our contributions in this paper to solve existing problems are presented in three-fold. Initially, we introduce a completely automatized hybrid method for segmenting the skin lesion by making use of K-means with Particle Swarm Optimization (PSO) technique. As far as we know, this proposed work is amongst the first couple of endeavors to use the concept of hybridization of PSO to tackle this challenging problem. Afterwards, we design an apt function for hair removal capable of naturally handling the lesion-background imbalance of pixel-wise classification for medical imaging segmentation. As per our results this hair removal function can better enhance the segmentation performance by removing the hair over the lesion region then apply feature extraction. So, the uniqueness of extracted feature is more for BCC and Non-BCC data. At end, we comprehensively evaluate the effectiveness, degree of efficiency and the generalizing ability of the proposed model with Dermoscopy Skin Lesion Image Dataset using ANN as a classifier. This proposed model can be easily generalized to other challenging medical image segmentation problems and also improve the accuracy of prognostics model for skin cancer detection and classification from dermoscopy images.

\section{STRUCTURE OF PROPOSED METHODOLOGY}

The proposed model for BCC detection and classification from dermoscopy images employing an artificial neural network consists of three main steps. Firstly, various image pre-processing techniques will be applied to enhance the image quality and suitable segmentation technique will be applied to separate out background from the image to extract exact Region of Interest (ROI). Here, to enhance the segmentation accuracy, PSO as an optimization algorithm is used to minimize the unwanted background region from ROI using the fitness function. Then features will be extracted from the segmented ROI lesion region and reduced further (if required) to feed as input to the ANN model. To extract the feature from ROI, SURF feature descriptor is used which helps to returns best key points from lesion ROI. Finally, recognition will be performed using ANN model to produce desired output for the automatic intelligent prognostics model. 


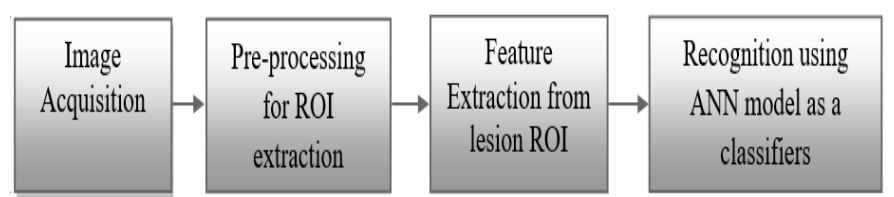

Fig. 3 : Block diagram of proposed model

The challenge of this research work is to detect and classify the Cell Carcinoma (BCC) using dermoscopy images and train the system using Artificial Neural Network (ANN) on the basis of the features extracted from the ROI of lesion using SURF descriptor. The subsequent steps demonstrate the variety of phases that need to be accomplished.

Data Acquisition: Upload the dermoscopy images of skin lesion database of different classes to train and test the system. In both section of model, training as well as testing skin lesion images is uploaded for processing. In training the number of images is more but in testing single test image is uploaded. The data uploading process is known as Skin Lesion Region Acquisition (SLRAQ) and the algorithm of SLRAQ is given as:

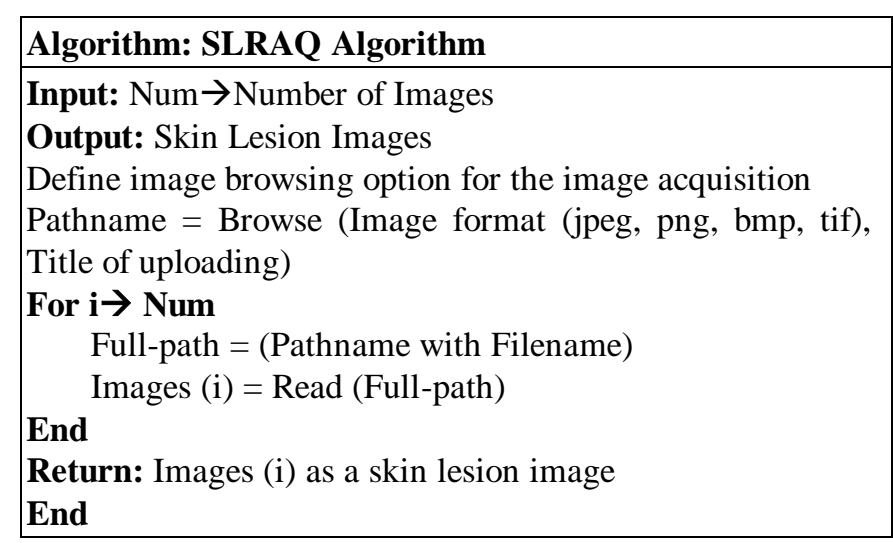

Pre-processing: Pre-Processing is done to remove various type of noise that are inherited in the skin lesion images and to enhance the image quality in proposed system. In every recognition system, selection of proper image region is the major factor and need to remove extra part from the images. In pre-processing after that, image segmentation is done to discover the region of interest (ROI) which helps to achieve better detection accuracy. For selecting the ROI from lesion images, PSO based K-means (Optimized K-means) is used which is based on the morphological operations like binarization, thinning, etc. Morphological operation is buildup of non-linear operations associated with the shape or morphology of features in an image. Morphological operations are applied on the binary image to find out the exact region of lesion within the image using some basic operations. There are a lot of morphological operations are available but used operations are given in the below figure.
Dilation - Grow text region

\section{Erosion - Shrink text region}

A Opening - Boundary pixels removal

\section{Closing - Boundary pixels filling}

\section{Thinning - To thin the text region}

Fig. 4: Morphological Operations

Fig. 4 represents the morphological operations which are used in the proposed intelligent prognostics model. In the proposed work, morphological operations help to find out the exact lesion region and separate the extra region from images. The used pre-processing steps are:

1. Image enhancement: Image enhancement is used to improve the specific pixel points of a dermoscopy image, rather than fix problems. In the proposed work, intensity based image enhancement (I2E) technique is used to improve the quality of image which helps to minimize the hair region from lesion so that the feature of skin lesion is appropriate. The $\mathrm{I} 2 \mathrm{E}$ algorithm is given as:

\begin{tabular}{|l|}
\hline Algorithm: I2E Algorithm \\
\hline Input: Gimg $\rightarrow$ Gray Image \\
Output: Eimg $\rightarrow$ Enhanced Image \\
I=double (Gimg) \\
[Height, Width, Plane] = size of I \\
Patch Size = P \\
Pad Size = p
\end{tabular}

Pmat $=$ Pad array (I, [Pad Size, Pad Size] $) / /$ It is blank mask according to the Pad Size

For $\mathbf{j} \rightarrow 1$ to Height

For $i \rightarrow 1$ to Width

Patch $=$ Pmat $(\mathrm{j}:(\mathrm{j}+$ PatchSize-1), $\mathrm{i}:(\mathrm{i}+$ patchSize-1),All $)$

$\operatorname{Zmat}(\mathrm{j}, \mathrm{i})=\min (\operatorname{Patch}(\mathrm{z}))$

End

End 


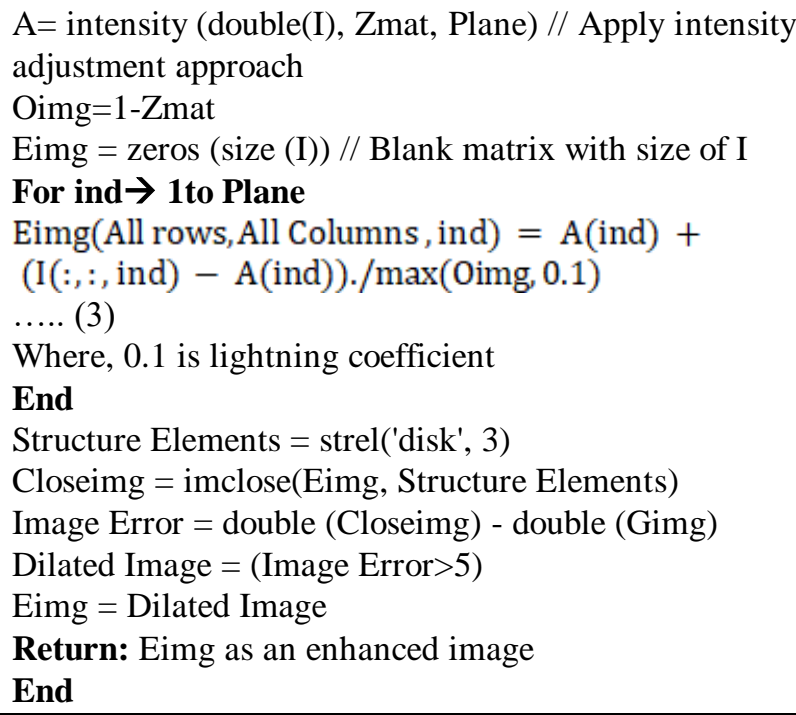

2. Binarization: The binarization process is carried out using the following equation with threshold value.

$$
\operatorname{Bimg}(i, j)=\left\{\begin{array}{l}
1 \text { ifEimg }(i, j) \geq \text { Threshold } \\
0 \quad \text { else }
\end{array} .\right.
$$

As shown in the equation (4), Bimg is binary image and Eimg is enhanced image with row (i) and columns (j). The binarization algorithm of proposed work is given as:

\begin{tabular}{|l|}
\hline Algorithm: Binarization Algorithm \\
\hline Input: Eimg $\rightarrow$ Enhanced Image \\
Output: Bimg $\rightarrow$ Binary Image \\
Define Row (R) and Columns (C) of Eimg \\
For $\mathbf{i}=\mathbf{1} \rightarrow \mathbf{R}$ \\
For $\mathbf{j}=\mathbf{1} \rightarrow \mathbf{C}$ \\
Using equation (4) \\
If Eimg(i, $\mathbf{j})>$ Average (Eimg) \\
Bimg(i,j) $=1$ \\
Else if Eimg(i, $\mathbf{j})<$ Average (Eimg) \\
Bimg $(i, j)=0$ \\
End \\
End \\
End \\
Return: Bimg as a binary image \\
End
\end{tabular}

Area Opening: The area opening is performed to remove the pixels from the boundary of lesion region in image so we can find out the well appropriate region. The opening is performed by using the given equation:

$$
\text { OpenImage }=\left\{\begin{array}{l}
0, \text { Pixels }<\text { Threshold } \\
1, \text { Pixels } \geq \text { Threshold }
\end{array}\right.
$$

Where, Pixels: Binary image pixels

Threshold: Threshold pixels value which helps to remove the boundary pixels

On the basis for morphological operations, Optimized $\mathrm{K}$-means is designed and the algorithm of Optimized $\mathrm{K}$-means is given as:

\section{Algorithm: Optimized K-means}

Input: SL Image $\rightarrow$ SkinLesion Image

Output: ROI $\rightarrow$ Skin LesionROI Image

$[R, C, P]=$ size (SL Image)

SL Image $=$ double $($ SL Image $)$

Number of Part $=2$

SimgIndex $=$ kmeans(SL Image, Number of Part)

SegLabelImg $=$ reshape $($ SimgIndex, R, C)

DataPos $=$ find (SegColLabelImg $>0$ )

Data $=$ SegColLabelImg(DataPos)

Initialize PSO parameter - Iterations (T)

- Swarm Size (S)

- Lower Bound (LB)

- Upper Bound (UB)

- Fitness function

Calculate $\mathrm{T}=$ Size (SL Image)

Fitness function:

$$
f(f i t)=\left\{\begin{array}{lr}
1 & \text { ifpixelisless } \\
0 & \text { otherwise }
\end{array}\right.
$$

For $\rightarrow \mathrm{T}$

$f s=\sum_{i=1}^{P} \operatorname{Data}(i)$

$f t=\frac{\sum_{i=1}^{P} \text { Data }(i)}{\text { Lengthoffeature }}$

$f(f i t)=$ fitness function which define by above given equation (6)

Threshold $_{\text {value }}=P S O(P, T, L B, U B, N, f(f i t))$

End

While $\mathbf{T} \sim=$ Maximum

Threshold $=$ Threshold $_{\text {value }}$

MaskImg=Morphological (SimgIndex, Threshold)

Boundaries $=$ bwboundaries (MaskImg)

Segmented Region $=$ Boundaries

For $i \rightarrow 1:$ P

Segmented Image $=$ SL Image X Segmented Region

End

Return; Segmented Image as ROI of Skin Lesion Image

End

After the Optimized K-means applied on the enhanced dermoscopy skin lesion images, we obtained below given results which are useful in next process of proposed work.

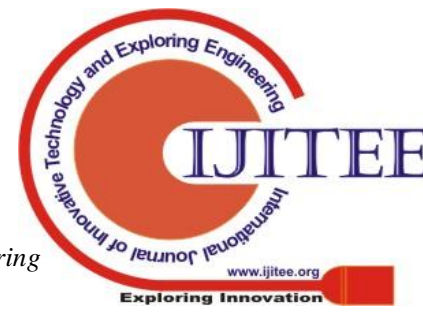




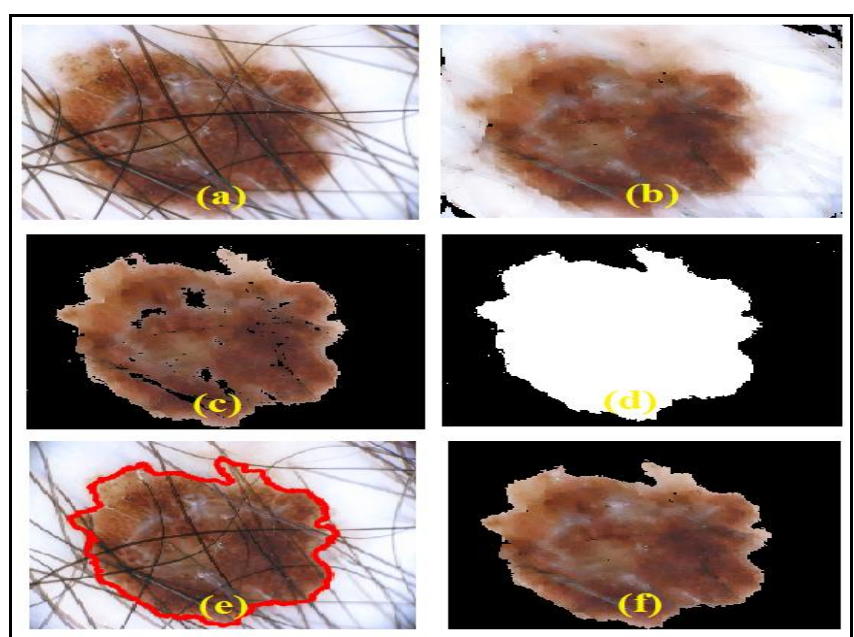

Fig. 5: ROI Extraction Process using Optimized K-means

Fig. 5 represent the ROI extraction process with pre-processing steps, where (a) is the original dermoscopy image, (b) is the enhanced dermoscopy image in which hair from lesion is removed, (c) presents the segmented image using K-means algorithm, (d) is the mask of lesion ROI, (e) is the region of lesion in dermoscopy image and (f) is the segmented ROI using the Optimized K-means algorithm. Form the figure, the achievement of proposed hybrid segmentation technique is represented and it helps in the proposed intelligent prognostics model for disease prediction and classification.

Feature Extraction: Extract feature from the ROI of lesion image based on the SURF feature extraction algorithm. After the feature extraction algorithm, a set of features is return by the SURF algorithm in terms of feature points. The SURF algorithm is given as:

\begin{tabular}{|l|}
\hline Algorithm: SURF Algorithm \\
\hline Input: ROI $\rightarrow$ ROI image \\
Output: Fpoints $\rightarrow$ Feature points \\
Load ROI data of lesion image \\
Calculate size of dataset [Row, Col.] \\
For $\mathbf{i} \rightarrow \mathbf{1}$ to all Row \\
For $\mathbf{j} \rightarrow \mathbf{1}$ to all Col \\
Scale image=scaling (ROI (i,j), scale size) \\
L_Keypoints=Localization (scale image (i,j)) \\
O_Keypoints=Orientation (L_Keypoint $(\mathrm{i}, \mathrm{j})$, Angle) \\
SURF_Keypoints=Filtering (O_Keypoint (i.j), Square Filter) \\
End \\
End \\
Return: Feature_descriptor as Fpoints \\
End
\end{tabular}

After the feature extraction algorithm applied on the lesion ROI, we obtained below given results which are useful in training as well as classification process of proposed work. To extract feature from lesion ROI, SURF Descriptor is used and applied on the lesion ROI. After the algorithm is applied on the ROI of lesion, the marked image with SURF point is given in the figure 6 . The extracted feature is passes to the ANN as an input training data and store which is used in the classification process to classify the diseases from the images of skin lesion which is taken from Dermoscopy Skin Lesion Image Dataset.

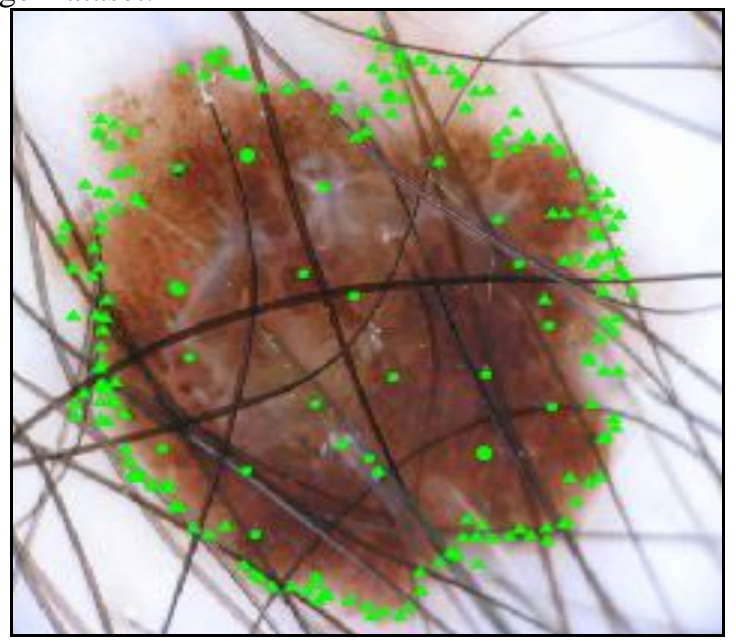

Fig. 6: SURF Feature of ROI

Training: Initialize ANN for classification purpose using two phases, namely, training and testing. After the training of system, we save the trained structure which is use in the classification section to classify the diseases from skin lesion images. In the testing phase, the test dermoscopy skin lesion image is uploaded and repeats the all steps. In the classification section, test skin lesion image SURF feature is matched with trained ANN structure and return disease type and the used ANN algorithm is given as:

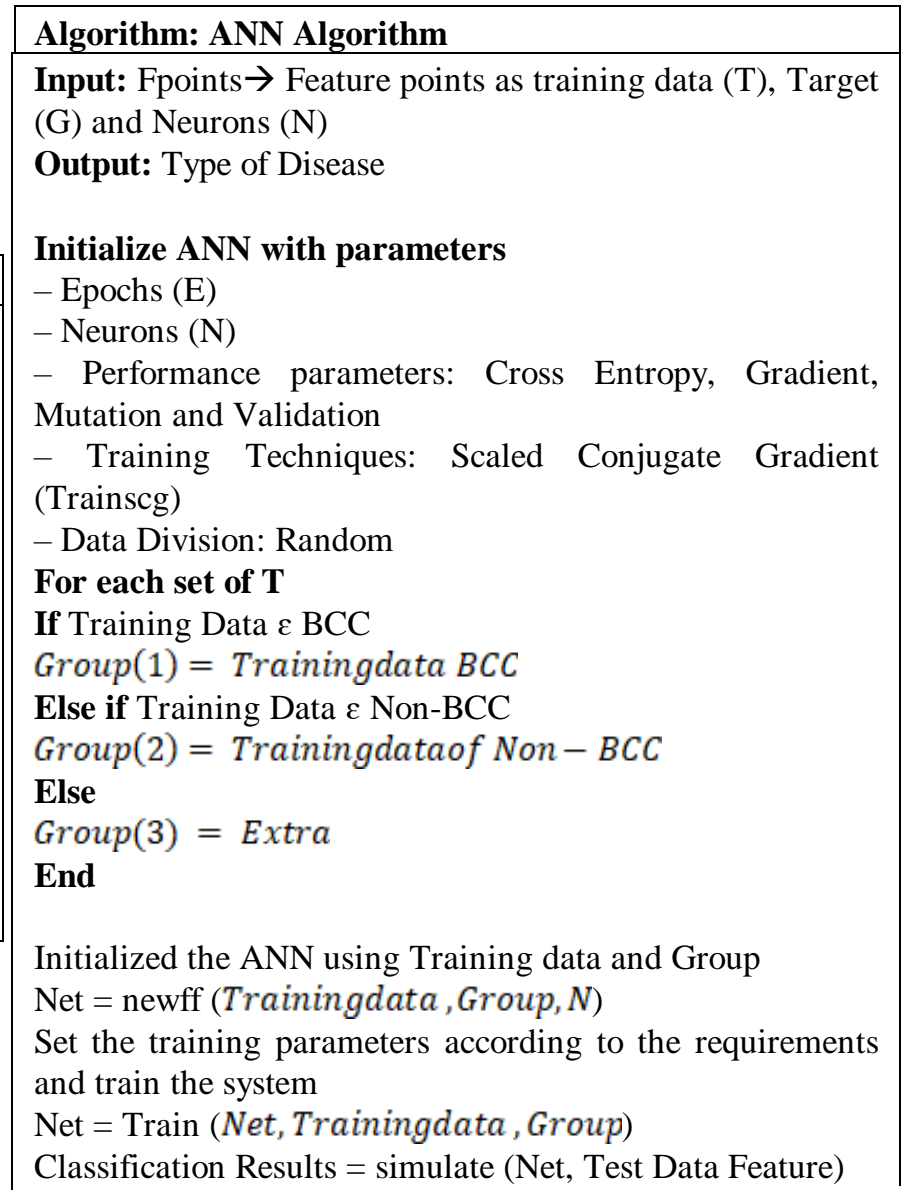

\section{If Classification Results =} True 
Show classified results in terms of the disease and predicted level of disease

Calculate the performance parameters

\section{End}

Return: Classified Results

End

Dermoscopy Skin Lesion Image Dataset: Dataset utilized is composed of images from two sources: 1) Atlas of Dermoscopy by Argenziano [14]; 2) ISIC Archive [15]. Images in dataset were acquired randomly where each image holds an equal chance of being selected as a part of the dataset. The diagnosis of these lesion images were given along with the images. Fig. 7 represents the samples of used dataset in proposed model for BCC prediction and classification using an artificial neural network based on the hybrid segmentation technique. In the database all images are dermoscopy image with jpg format and in the image dataset any type of compression is not applied. All images are non-compressed and electronic noise free. In the dataset, mainly two types of categories of skin lesion are presents, first is BCC and another is Non-BCC. Types of BCC images are also called as malignant type.

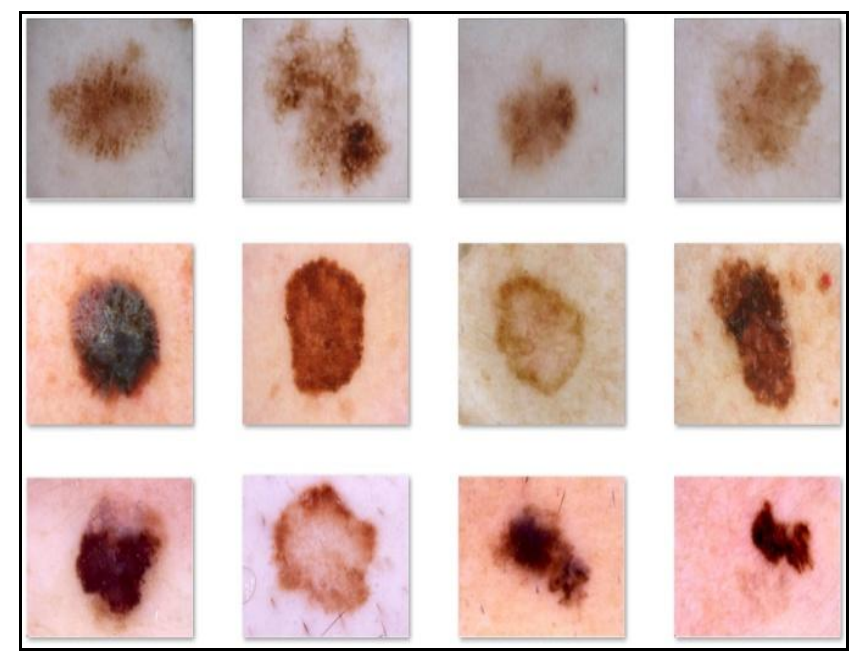

Fig. 7: Dataset Sample of Proposed Work

The flowchart of the proposed work is shown in Fig. 8 with training and testing process with Dermoscopy Skin Lesion Image Dataset.

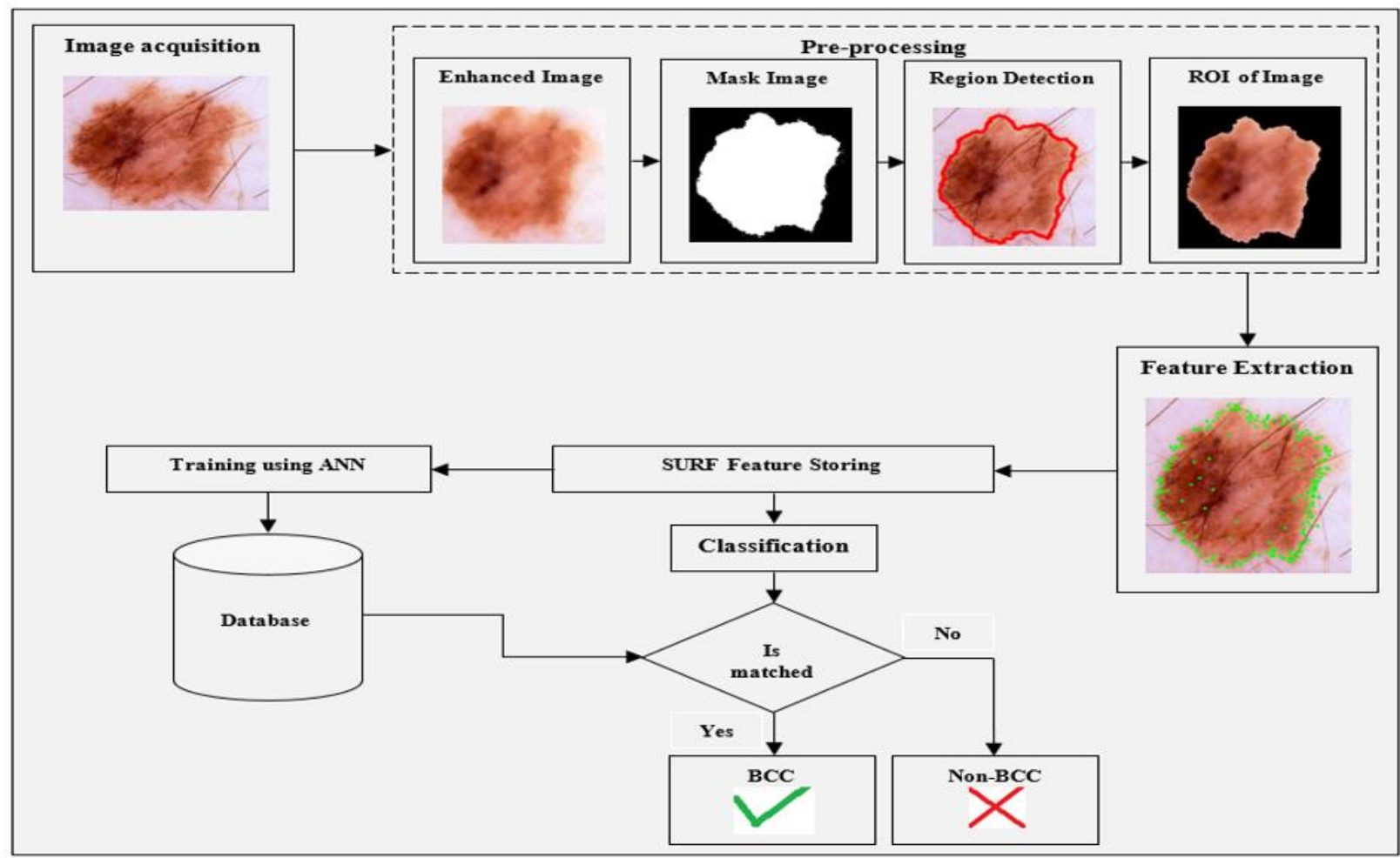

Fig. 8 : Flowchart of proposed work

\section{RESULTS AND DISCUSSIONS}

This section discusses the simulation results of proposed intelligent prognostics model and compares the efficiency of proposed work with existing work [2]. The training and testing of the proposed mechanism is evaluated by Dermoscopy Skin Lesion Image Dataset. By adapting the established proposed algorithms, below outcomes are computed with quality based parameters, in particular, True Positive Rate, False Positive Rate, Precision and Accuracy in terms of Area Under Curve (AUC). A comparison is drawn by randomly taking three test samples namely TS 1 , TS 2 , TS 3 versus the existing work [2] to show the effectiveness of the proposed work with respect to BCC and Non-BCC. 
Improved Segmentation algorithm using PSO and K-means for Basal Cell Carcinoma Classification from Skin Lesions

Table 1 : Test results of proposed method

\begin{tabular}{|c|c|c|c|c|c|}
\hline Types & Test Sample & TP Rate & FP Rate & Precision & AUC \\
\hline \multirow{3}{*}{ BCC } & TS 1 & 0.935 & 0.0383 & 0.983 & 0.973 \\
\cline { 2 - 6 } & TS 2 & 0.923 & 0.0484 & 0.979 & 0.981 \\
\cline { 2 - 6 } & TS 3 & 0.981 & 0.0234 & 0.986 & 0.976 \\
\hline \multirow{3}{*}{ Non-BCC } & TS 1 & 0.948 & 0.0459 & 0.937 & 0.985 \\
\cline { 2 - 6 } & TS 2 & 0.953 & 0.0386 & 0.963 & 0.978 \\
\cline { 2 - 6 } & TS 3 & 0.985 & 0.0265 & 0.982 & 0.976 \\
\hline
\end{tabular}

Table 2: Test results of existing method [2]

\begin{tabular}{|c|c|c|c|c|}
\hline Types & TP Rate & FP Rate & Precision & AUC \\
\hline BCC & 0.859 & 0.061 & 0.914 & 0.965 \\
\hline Non-BCC & 0.939 & 0.141 & 0.898 & 0.965 \\
\hline
\end{tabular}

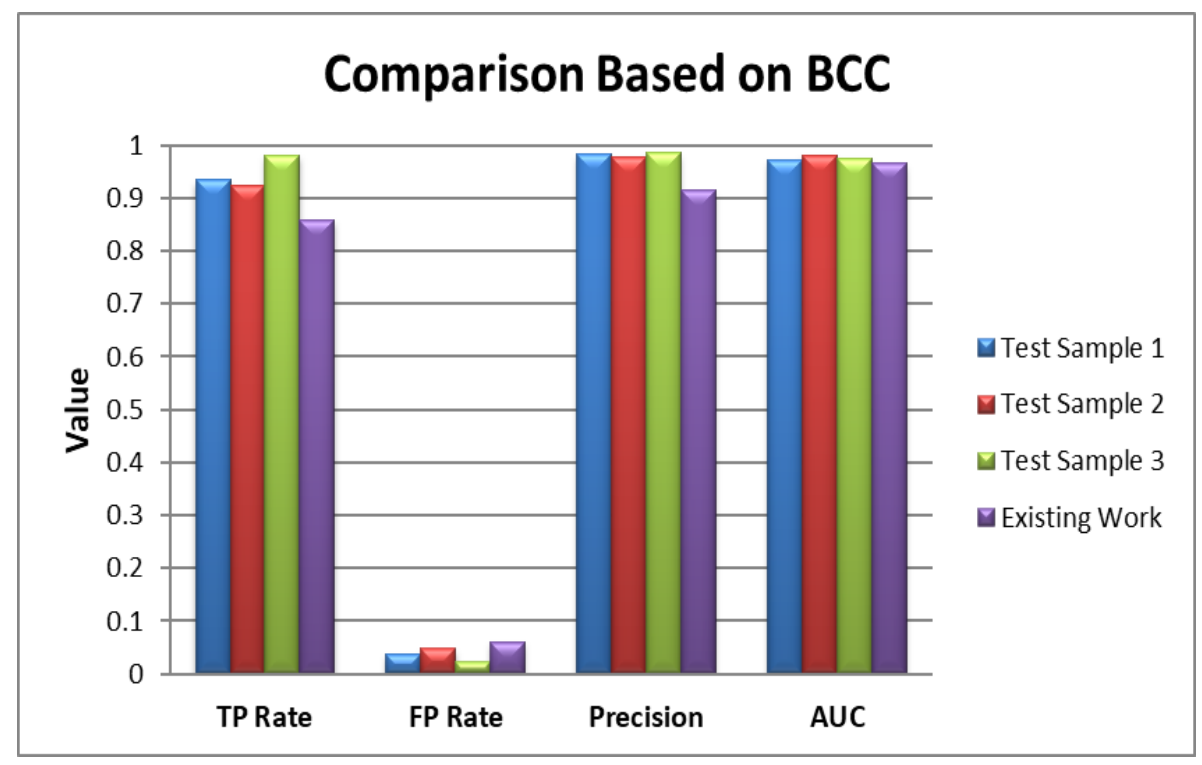

Fig. 9: Comparison of evaluation parameters based on BCC

The comparison of evaluation parameters for proposed work test samples and existing work is depicted in Fig. 9 for BCC data, where average TP Rate is 0.946 , FP Rate is 0.0367, Precision is 0.983 and Accuracy (AUC) is 0.976 for the proposed work.
TP Rate is the probability of accurately enrollment of features which are included in the matching process and FP Rate is the rate of erroneously accepted feature during classification. Precision is the rate of TP and summation of TP and FP which represents the accuracy of participating feature of classified category of disease. 


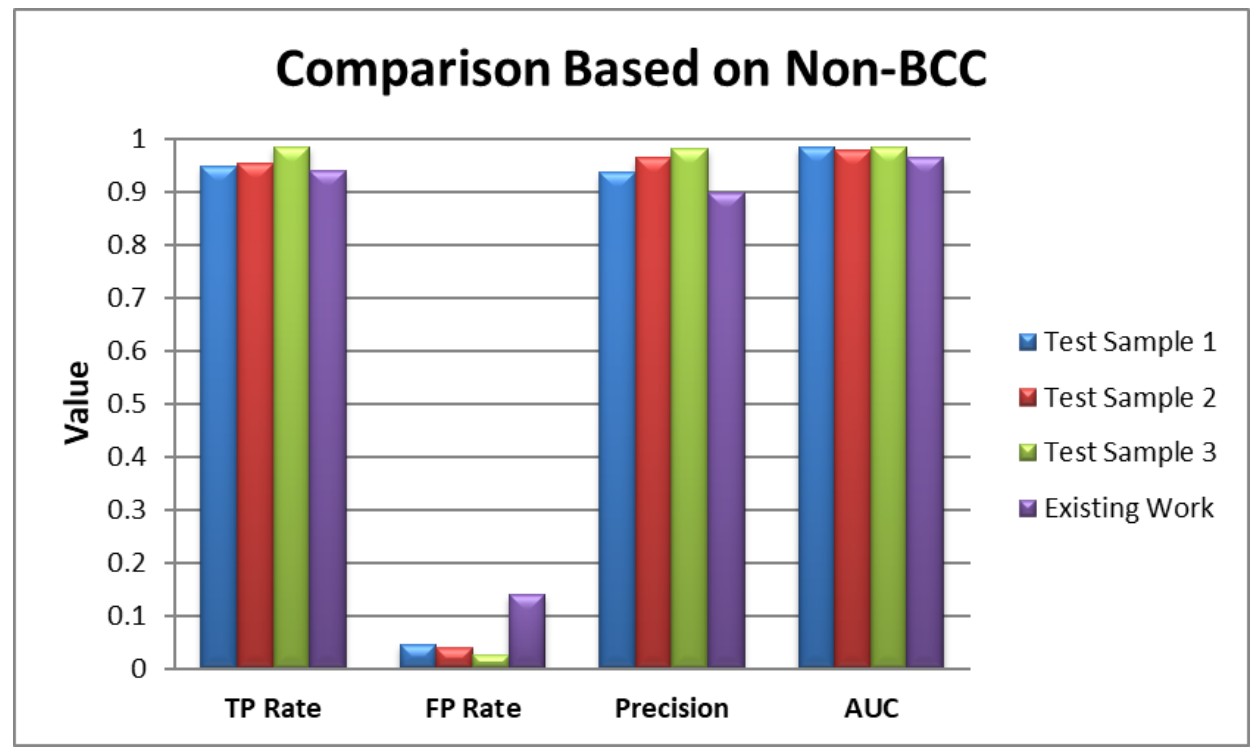

Fig. 10: Comparison of evaluation parameters based on Non-BCC

The comparison of evaluation parameters for proposed work test samples and existing work is depicted in Fig. 10 based on the Non-BCC data, where average TP Rate is 0.962 , FP Rate is 0.037 , Precision is 0.961 and Accuracy (AUC) is 0.982 for the proposed work.
From the above observation, we conclude that the accuracy of proposed work is superior to existing work for BCC as well as Non-BCC data. The comparison of proposed approach with some other existing approaches, which is considered in survey of proposed work, is described in Table 3.

Table 3: Comparison of accuracy of proposed work with existing works

\begin{tabular}{|l|c|}
\hline \multicolumn{1}{|c|}{ Authors } & Accuracy (\%) \\
\hline Pegah Kharazmi [2017] & 96.5 \\
\hline Lequan Yu [2017] & 93.1 \\
\hline Yading Yuan [2017] & 96.3 \\
\hline N. C. F. Codella [2017] & 71.5 \\
\hline FengyingXie [2017] & 91.1 \\
\hline Proposed work & 97.9 \\
\hline
\end{tabular}




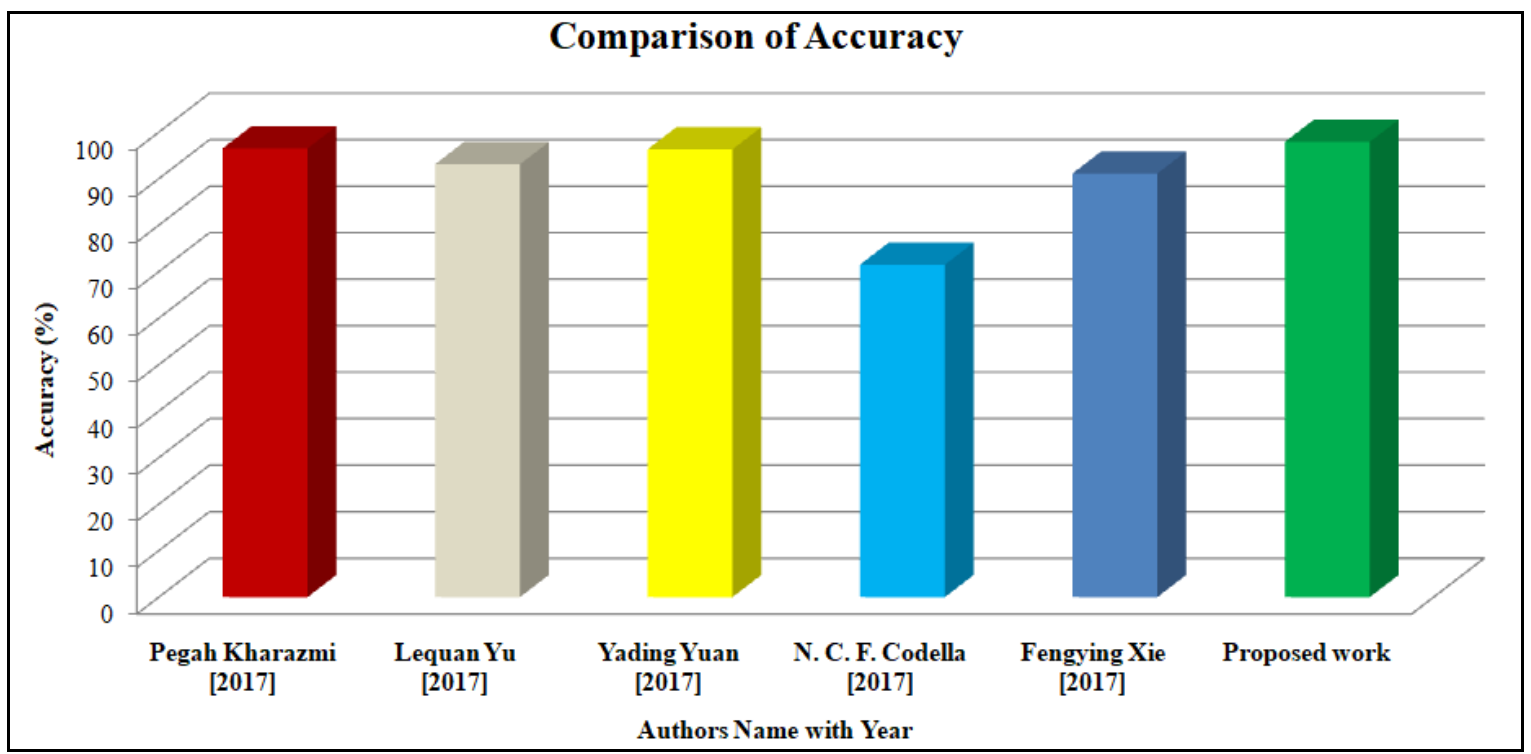

Fig. 11: Comparison of accuracy of proposed work with existing works

Fig. 11 represents the comparative analysis of existing work based on the classification accuracy. It clearly illustrates that the accuracy achieved by proposed work is better than other authors by using the hybrid segmentation with the concept of ANN using SURF feature extraction technique.

However, this proposed research work has a few restrictions. It isn't appropriate for images having more hairs and the segmentation accuracy is exceptionally low for such hairy skin lesion images. Besides, the time complexity of the system is quite high for expansive datasets amid the training phase.

\section{CONCLUSION AND FUTURE WORK}

In this paper, an improved approach for BCC prediction and classification from dermoscopy images using an artificial neural network based on the hybrid segmentation technique is proposed. It bestows a comprehensive description of the different applications and potential challenges of segmentation and classification of BCC from skin lesions which is a difficult task in medical science. For the detection and classification of skin diseases, segmentation of skin lesions is major task and it is performed by hybridization of K-means with PSO. After that, in this paper we present an ANN with SURF descriptor for the segmentation and classification of BCC and Non-BCC data. Utmost classification accuracy is reported when proposed work is simulated on dataset using the concept of ANN. With proposed method, the accuracy is $97.9 \%$ whereas the accuracy of the existing work is less.
In future work, ANN can be used as a classifier to train system based on hybridization of SURF descriptor with soft computing based feature selection algorithm.

\section{REFERENCES}

1. http://cancerhelpessentiahealth.org/images/cdr/live/CDR579033-7 50.jpg.

2. P. Kharazmi, M. I. AlJasser, H. Lui, Z. J. Wang and T. K. Lee, "Automated detection and segmentation of vascular structures of skin lesions seen in Dermoscopy, with an application to basal cell carcinoma classification." IEEE journal of biomedical and health informatics vol. 21, no.6, pp. 1675-1684, 2017.

3. L. Yu, H. Chen, Q. Dou, J. Qin and P. Heng, "Automated melanoma recognition in dermoscopy images via very deep residual networks", IEEE transactions on medical imaging, vol. 36, no.4, pp. 994-1004, 2017.

4. Y. Yuan, M. Chao and Y. Lo, "Automatic skin lesion segmentation using deep fully convolutional networks with jaccard distance", IEEE Trans. Med. Imaging, vol. 36, no. 9, pp. 1876-1886, 2017.

5. N. C. F. Codella, Q. B. Nguyen, S. Pankanti, D. A. Gutman, B. Helba, A. C. Halpern and J. R. Smith, "Deep learning ensembles for melanoma recognition in dermoscopy images." IBM Journal of Research and Development, vol. 61, no. 4/5, pp. 5:1-5:15, 2017.

6. F. Xie, H. Fan, Y. Li, Z. Jiang, R. Meng and A. Bovik "Melanoma classification on dermoscopy images using a neural network ensemble model", IEEE transactions on medical imaging, vol. 36, no.3, pp. 849-858, 2017.

7. E. Ahn, J. Kim, L. Bi, A. Kumar, C. Li, M. Fulham and D. D. Feng, "Saliency-based lesion segmentation via background detection in dermoscopic images", IEEE journal of biomedical and health informatics, vol. 21, no. 6, pp. 1685-1693, 2017.

8. C. Benazzi, A. Al-Dissi, C. H. Chau, W. D. Figg, G. Sarli, J. T. de Oliveira and F. Gartner, "Angiogenesis in spontaneous tumors and implications for comparative tumor biology", The Scientific World Journal, 2014.

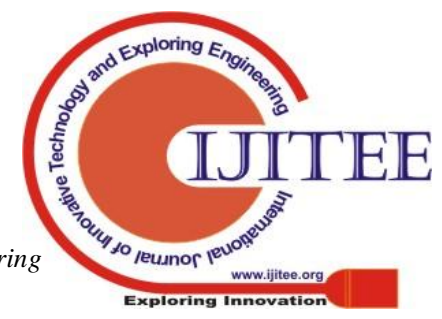


9. B. Cheng, R. J. Stanley, W. V. Stoecker, and K. Hinton, “Automatic telangiectasia analysis in dermoscopy images using adaptive critic design”, Skin Research and Technology, vol. 18, pp. 389-396, 2011.

10. J. W. Choi, B. R. Kim, H. S. Lee, and S. W. Youn, "Characteristics of subjective recognition and computer-aided image analysis of facial erythematous skin diseases: A cornerstone of automated diagnosis", Brit. J. Dermatol., vol. 171, pp. 252-258, 2014.

11. S. C. Hames, S. Sinnya, J-M Tan, C. Morze, A. Sahebian, H. P. Soyer, T. W. Prow, "Automated Detection of Actinic Keratoses in Clinical Photographs", PLoSONE10(1): e0112447, doi:10.1371/journal. pone.0112447, 2015.

12. B. Cheng, R. J. Stanley, W. V. Stoecker, S. M. Stricklin, K. A. Hinton, T. K. Nguyen, R. K. Rader, H. S. Rabinovitz, M. Oliviero and R. H. Mos., "Analysis of clinical and dermoscopic features for basal cell carcinoma neural network classification," Skin Research and Technology, vol. 19, pp. e217-e222, 2012.

13. P. Kharazmi, H. Lui, W. V. Stoecker, and T. Lee, "Automatic detection and segmentation of vascular structures in dermoscopy images using a novel vesselness measure based on pixel redness and tubularness", Proc. SPIE, vol. 9414, Computer-Aided Diagnosis, 94143M, 2015.

14. H. S. G. Argenziano et al., Interactive Atlas of Dermoscopy, Milan, Italy: Edra Medical Publishing and New Media, 2000. [Online]. Available: https://sites.google.com/site/robustmelanomascreening/dataset.

15. International Skin Imaging Collaboration (ISIC) archive. [Online]. Available: https://isic-archive.com. 